A. Pramesh Rao, G. Swarup and Gopal-Krishna, eds.

\title{
Radio Frequency Interference
}

\author{
R. D. Ekers and J. F. Bell \\ ATNF CSIRO, PO Box 76 Epping NSW 1710, Sydney, Australia
}

\begin{abstract}
We describe the nature of the interference challenges facing radio astronomy in the next decade. These challenges will not be solved by regulation only, negotiation and mitigation will become vital. There is no silver bullet for mitigating against interference. A successful mitigation approach is most likely to be a hierarchical or progressive approach throughout the telescope and signal conditioning and processing systems. We summarise some of the approaches, including adaptive systems.
\end{abstract}

\section{The RFI Challenge and Spectrum Management}

If future telescopes like the SKA are developed with sensitivities up to 100 times greater than present sensitivities, it is quite likely that current regulations will not provide the necessary protection against interference. There is a range of experiments (eg redshifted hydrogen or molecular lines) which require use of arbitrary parts of the spectrum, but only at a few locations, and at particular times, suggesting that a very flexible approach may be beneficial. Other experiments require very large bandwidths, in order to achieve enough sensitivity. As shown in Figure 1, presently only $1-2 \%$ of the spectrum in the metre and centimetre bands is reserved for passive uses, such as radio astronomy (Morimoto 1993). In the millimetre band, much larger pieces of the spectrum are available for passive use, but the existing allocations are not necessarily at the most useful frequencies. Current regulations alone will be inadequate, we need technology as well as regulation. We cannot (and do not want to) impede the telecommunications revolution, but we can try to minimise its impact on passive users of the radio spectrum and maximise the benefits of technological advances. Further information on many of the topics discussed below is available on http://www.atnf.csiro.au/SKA/intmit/.

\section{Classes of Interference}

It is important to be clear of what we mean when we talk about interference. Radio astronomers make passive use of many parts of the spectrum legally allocated to communication and other services. As a result, many of the unwanted signals are entirely legal and legitimate. We will adopt the working definition that interference is any unwanted signal entering the receiving system.

Interfering signals vary a great deal in their source and nature. This naturally leads to different mitigation approaches. Local sources of interference 

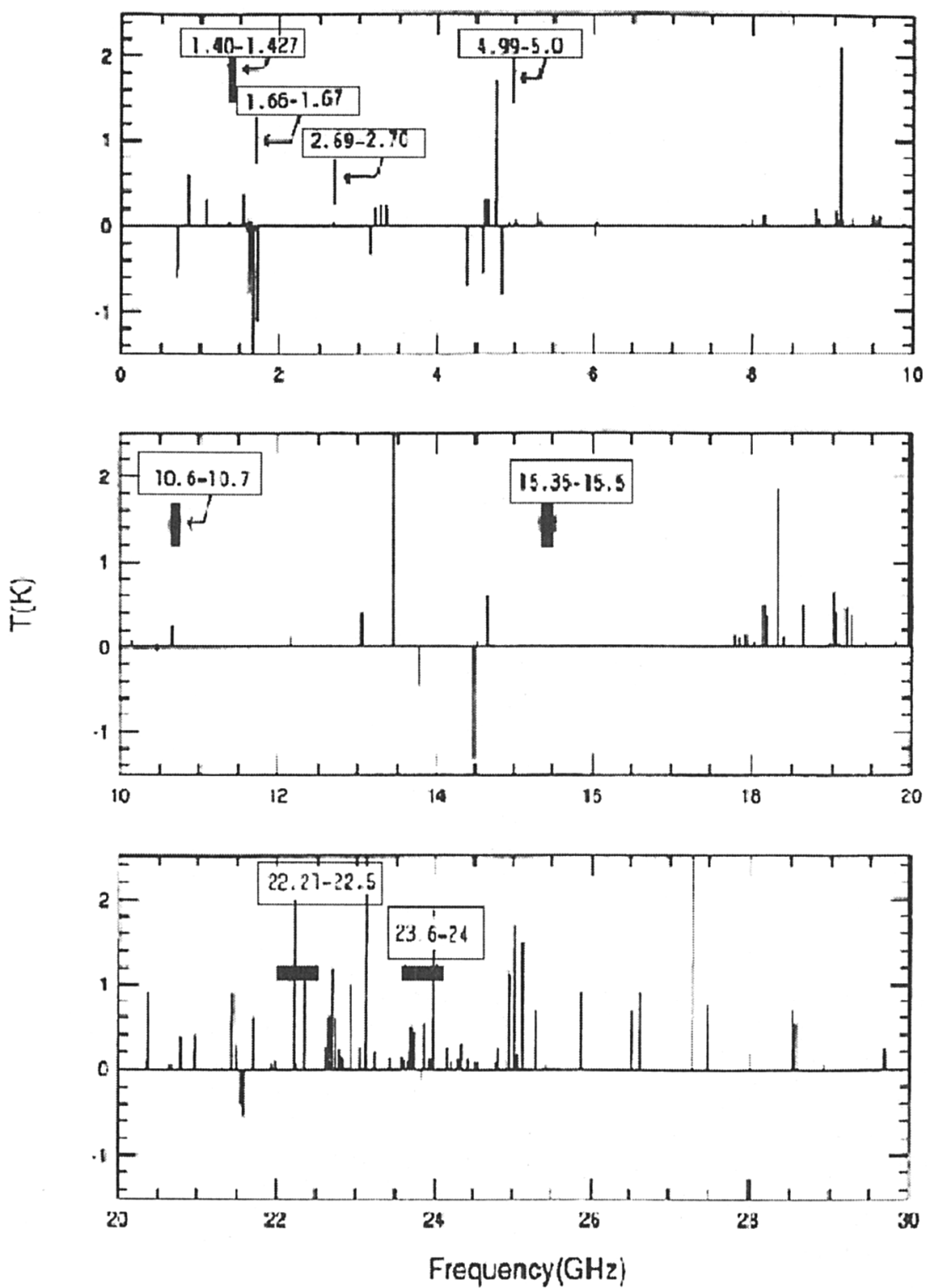

Figure 1. Spectral lines (at zero redshift) are indicated in absorption or emission over the $0-30 \mathrm{GHz}$ band. The boxes indicate the bands allocated for passive radio astronomy uses. Figure from Morimoto 1993. 
include things internal to telescope instruments, networking for IT systems, and general and special purpose digital processors in the observatory. Interference compliance testing, shielding, separate power circuits, minimising nearby equipment are key steps that need to be taken to minimise this kind of interference.

External interference may arise from fixed or moving sources. Not all methods of mitigation apply to both: in fact methods that work well for fixed sources, may not work at all for moving sources, due to problems like side lobe rumble. Interference may be naturally occurring or human generated. Examples of naturally occurring interference include: the ground, sun, other bright radio sources, lightening, and meteors. Human generated interference may come from broadcast services (eg TV, radio), voice and data communications (eg mobile telephones, two-way radio, wireless IT networks), navigation systems (eg GPS, GLONASS), radar, remote sensing, military systems, electric fences, car ignitions, and domestic appliances (eg microwave ovens) (Goris 1998).

The vast majority of these operate legally with in their allocated bands, regulated by national authorities and the ITU (International Telecommunications Union). However there are sources of interference, such as the Iridium mobile communications systems, whose signals leak into bands protected for passive use. In this case, these interfering signals are $10^{11}$ times stronger than the signal from the early universe. In the case of Australia, there is a single communications authority for whole country and therefore for the whole continent. As a result there is a single database containing information on the frequency, strength, location, etc of every licensed transmitter (Sarkissian 2000). A key point therefore, is that the modulation schemes and other characteristics of the vast majority of these signal are known. Their effect on radio telescopes is not only predictable, but can be modelled and used to excise the unwanted signals.

Radio astronomy could deal with most terrestrial interfering signals, by moving to a remote location, where the density and strength of unwanted signals is greatly reduced. As shown in Figure 2, this is getting more and more difficult, but there are still some possibilities. However with the increasing number of space borne telecom and other communications systems in low (and mid) Earth orbits, a new class of interference mitigation challenges are arising - radio astronomy can run, but it cannot hide! The are several new aspects introduced into the interference mitigation problem by this and they include: rapid motion of the transmitter on satellites, more strong transmitters in dish side lobes and possibly in primary beam, and different spectrum management challenges, because no place on Earth is free from interference from the sky.

\section{RFI fundamentals}

Undesired interfering signals and astronomy signals can differ (be orthogonal) in a range of parameters, including: frequency, time, position, polarisation, distance, coding, positivity, and multi path. It is extremely rare that interfering and astronomy signals do not possess some level of orthogonality in this $\geq 8$ dimensional parameter space. We therefore need to develop sufficiently flexible signal processing systems to take advantage of the orthogonality and separate the signals. This is of course very similar to the kinds of problems faced by mobile communication services, which are being addressed with smart antennas and 


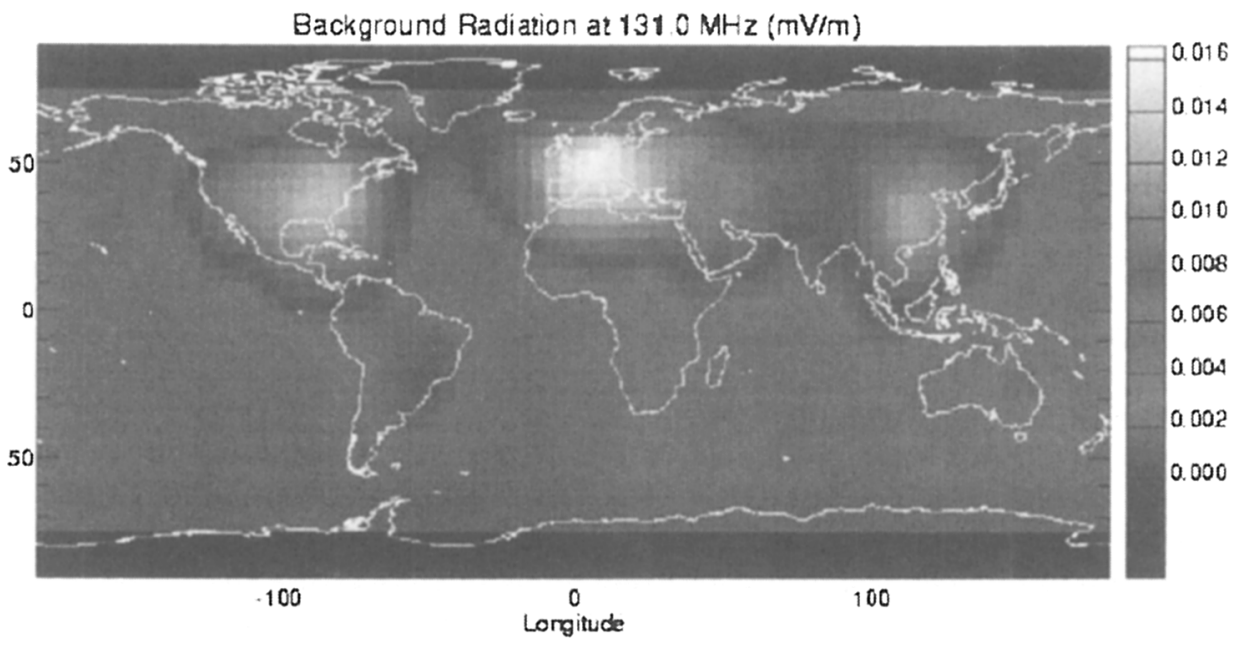

Figure 2. Forte satellite: $131 \mathrm{MHz}, \mathrm{RF}$ background data for calibration of lightening monitors provides a map of signals emanating from the Earths surface, many of which are human generated. Acquired by the Los Alamos National Laboratory FORTE spacecraft, Principal Investigator A. R. Jacobson. Data courtesy of R. J. Strangeway, UCLA.

software radio technologies. Examples in radio astronomy to date include the use of the time and frequency phase space in pulsar studies, and the requirement that signals are positive in very low frequency studies. Antenna arrays could take advantage of the position and distance (curvature of wavefront) phase space. Human generated interference is normally polarised, so unpolarised astronomical signals can be observed by measuring the unpolarised component $\left(I-\left(U^{2}+Q^{2}+\right.\right.$ $\left.\left.V^{2}\right)^{1 / 2}\right)$.

\subsection{Mitigation Strategies and Issues}

There is no silver bullet for detecting weak astronomical signals in the presence of strong undesired naturally occurring or human generated signals. Spectral bands allocated for passive use provide a vital window, which cannot be achieved in any other way. It is important to characterise the RFI so that the number, strength, band width, duty cycle, spatial and frequency distributions, and modulation and coding schemes can all be used to advantage in modelling and mitigating RFI. Doing this at low frequencies gives greater sensitivity due to the effects of harmonic content and ease of propagation. In order to do this, the telescope and instruments must be calibrated to provide the best possible characterisation of interfering and astronomy signals. A/D converters must be fast enough to give sufficient bandwidth, with a sufficient number of bits so that both strong and weak signals are well sampled. There are a range of techniques that can make 
passive use of other bands possible and in general these need to be used in a progressive or hierarchical way.

- Remove at source is obviously best, but that is often not possible,

- Regulation providing radio quiet frequencies or regions,

- Negotiation with owners users can lead to win-win solutions, for example replacing nearby radio links with underground fibres, removes interference and improves voice and data connectivity for users,

- Avoid interference by choosing appropriate locations with terrain screening or radio quiet zones,

- Move to another frequency,

- Screening to prevent signals entering the primary elements of receivers,

- Far Side lobes of primary and secondary elements must be both minimised and well characterised,

- Minimise coherent signals through out the array and thereby allow the natural rejection of the array to deal with the incoherent signals,

- Front end filtering, using for example high temperature super conducting filters with high $Q$ to reject strong signals in narrow bands, before they cause saturation effects.

- High dynamic range linear receivers to allow appropriate detection of both astronomy (signals below the noise) and very strong interfering signals,

- Notch filters (analog, digital or photonic) to excise bad spectral regions,

- Clip samples from data streams to mitigate burst type interference,

- Decoding to remove signals with complex modulation and multiplexing schemes. Blanking of period or time dependent signals is a very successful but simple case of this more general approach,

- Cancellation of undesired signals, before correlation using fixed and adaptive signal processing (harris, 2000),

- Post correlation cancellation of undesired signals, taking advantage of phase closure techniques (Sault 2000)

- Parametric techniques allow the possibility of taking advantage of known interference characteristics to excise it (Ellingson, 2000),

- Adaptive beam forming to steer one or more nulls onto interfering sources. This is equivalent to cancellation, but it provides a way of taking advantage of the spatial orthogonality of astronomy and interfering signals,

- Use of Robust statistics in data processing to minimise the effects of outliers.

\subsection{Which signal processing regime: traditional analog, digital, pho- tonic ?}

In most applications of signal processing, there is a strong trend towards the use of digital techniques, as well as photonic techniques. The fundamental reason for this is that digital and photonic devices have cost curves which are evolving much more rapidly than traditional analog systems. In addition to that, they open up new techniques and offer substantial reduction in computational effort in many cases. The inherent immunity of photonic approaches to radio interference also creates functional advantages (Minasian 2000). Astronomers are joining these trends for exactly the same reasons. The jury is still out on what the appropriate balance or mixture of these techniques will be. 


\section{Adaptive EMI rejection}

Adaptive rejection algorithms can be either constrained or unconstrained. Constrained algorithms generally incorporate either a model or a copy of the desired or interfering signal, which is used control the adaption. For example it may be constrained so that only those signals with a certain coding or chip sequence are removed (Ellingson, 2000). Most astronomy signals are expected to be pure noise, so one could envisage a constraint that rejects all non noise like signals. In the case of unconstrained adaption, some algorithms (predictive adaptive algorithms) simply assume that the interference is much stronger than the signal and just use previous data samples to predict the following data samples for cancellation (harris, 2000). If that assumption cannot be made, another approach is to block the desired signal and let the unconstrained algorithm work on the remaining signals. This is often done using a blocking matrix, which can be thought of as an operator that applies a set of complex weights which block certain signals, while passing everything else. Advantages of this approach are that it can deal with multiple interfering signals which are changing in time and space, without affecting the signal to noise of the desired signal.

A key ingredient of constrained adaptive algorithms is a reference channel that maximises interference to noise ratio for the ensemble of interferers. One way of achieving this is using additional omni-directional antennas or arrays which at least matches the gain of the side lobe response of the main array.

\subsection{Adaptive Interference Cancelling}

Of all the approaches listed above, the nulling or cancellation systems (may be adaptive or predictive) are the most likely to permit the observation of weak astronomy signals that are coincident in frequency or space with undesired signals. There is an important space-time duality with cancelling algorithms. Any algorithm that works in the time domain can also be applied in the space domain.

These techniques have been used extensively in communications, sonar, radar, medicine and others (Widrow \& Stearns 1985, Haykin 1995). Radio astronomers have not kept pace with these developments and in this case need to infuse rather than diffuse technology in this area. A prototype time based cancellation system developed at NRAO (shown in Figure 3) has demonstrated $70 \mathrm{~dB}$ of rejection on the lab bench and $30 \mathrm{~dB}$ of rejection on real signals when attached to the 140 foot at Green Bank (Barnbaum \& Bradley 1998). Adaptive nulling systems are being prototyped by NFRA in the Netherlands (van Ardenne, these proceedings). Combined space-time approaches have been used to cancel interference in GPS receivers (Trinkle, 2000). However, in all cases, the application in the presence of real radio astronomy signals is yet to be demonstrated and their effects on the weak astronomy signals needs to be quantified. A good prospect for doing this in the near future is recording baseband data from existing telescopes, containing both interfering and astronomy signals and simulating the receiver system in software (Bell et al. 1999). A number of algorithms can then be implemented is software and assessed relative to each other.

Beam forming and adaptive nulling as are not necessarily being done sequentially, but rather in parallel. While there are some sequential schemes (genetic algorithms for example), most approaches simultaneously solve for the 


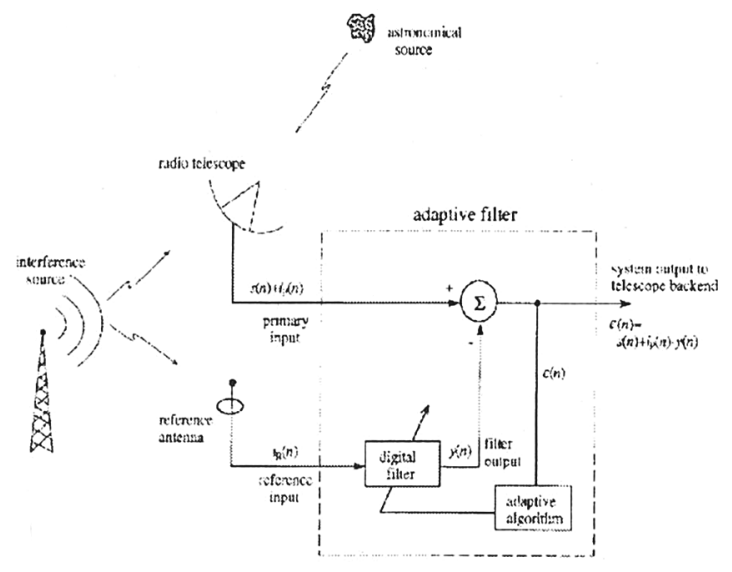

Figure 3. A Conceptual View of Adaptive Interference Cancelling. From Barnbaum \& Bradley (1998).

coefficients that give the desired beams and nulls. One can think of this as an optimisation problem. For example, in the minimum variance beam former, the "goal" is to minimise output power, and the "constraint" is maintain constant gain in a certain direction. The goal forces the nulls onto all interfering signals not coming from the direction of the astronomical source, and the constraint protects the beam gain (Ellingson, 2000). Of course, it is only protected for one direction, so there is still shape distortion. In general for an $\mathrm{N}$ element array, you can form up to $\mathrm{N}-1$ nulls. However if more control over the main beam shape is required, one may use other beam formers, which form a smaller number of nulls, and use more degrees of freedom to control the main beam shape.

A physical interpretation of why you can form nulls without wrecking the beam might go as follows: Phased arrays with many elements (not equally spaced), have lots of nulls, and they are all over the sky once you get a reasonable distance from the main beam. Imagine changing the coefficients a little to get the closest one on to an interferer. Very little variation in the coefficients is required. Since the difference is so small, the main beam hardly notices. The other nulls will shift around, of course, because they are sensitive to small changes in the coefficients. Close to the main beam, the nulls are further apart, so you need a bigger variation in the coefficients to nudge the closest null into place - hence the increased distortion in the main beam in this case. It may be necessary to record the weights applied to generate the nulls, so that the beam shape changes can be calibrated out later (Cram, 2000). 


\section{Real time $v$ post correlation}

Real time systems permit full recovery of temporal information in the signal required. Real time systems have been well studied and numerous examples can be found in other fields such as: radar, sonar, communications, defence anti jam, speech processing, and medicine. For example, there are existing systems which are capable of nulling up to 7 simultaneous moving jammers. In radio astronomy we operate in a totally different regime in which the astronomical signals are weak and noise like. We only wish to measure the time averaged statistical properties of the signals. For example in aperture synthesis the time averaged coherence between two antennas. Since we don't have to recover the signal modulation, radio astronomy does not have to use the real time algorithms developed for communications and radar. In such post correlation systems (Sault 2000 ) the information is only contained in the statistical properties of the signal which may vary slowly in time, frequency, space or direction. Both the signal of interest and the interference obey phase and amplitude closure relations. This results in an over determined set of equations which form a closed set which can be used to self calibrate the array for both the source and the interferer.

We conjecture that: "post correlation processing of time averaged signals can achieve the same RFI rejection as in real-time algorithms and that self calibration (phase closure) techniques provide powerful additional constraints".

\section{References}

Barnbaum, c. \& Bradley, R., 1998, AJ, 116, 2598.

Bell J., et al. 1999 "Software radio telescope: interference mitigation atlas and mitigation strategies", in Perspectives in Radio Astronomy: Scientific Imperatives at $\mathrm{cm}$ and $\mathrm{m}$ Wavelengths (Dwingeloo: NFRA), Edited by: M.P. van Haarlem \& J.M. van der Hulst.

Cram, L., 2000, "System Considerations", RFI Mitigation Strategies. http://www.atnf.csiro.au/SKA/intmit/atnf/conf/

Ellingson, S., 2000, "Interference Mitigation techniques", RFI Mitigation Strategies. http://www.atnf.csiro.au/SKA/intmit/atnf/conf/

harris, f., 2000, "RFI Mitigation", RFI Mitigation Strategies. http://www.atnf.csiro.au/SKA/intmit/atnf/conf/

Goris, M., 1998, "Categories of Radio Interference", NFRA Technical Report 415/MG/V2.3, http://www.nfra.nl/skai/archive/technical/index.shtml

Haykin, S., 1995, "Adaptive Filter Theory" Prentice Hall.

Minasian, R., 2000, "Photonics in radio astronomy", RFI Mitigation Strategies. http://www.atnf.csiro.au/SKA/intmit/atnf/conf/

Morimoto, M., 1993, "Charm \& Crisis in Radio Astronomy", Modern Radio Science.

Sarkissian, J., 2000, "Transmitter database visualisation", RFI Mitigation Strategies. http://www.atnf.csiro.au/SKA/intmit/atnf/conf/

Sault, R., 2000, "Synthesis Arrays", RFI Mitigation Strategies. http://www.atnf.csiro.au/SKA/intmit/atnf/conf/

Trinkle, M., 2000, "A DSP testbed for interference mitigation in GPS", RFI Mitigation Strategies. http://www.atnf.csiro.au/SKA/intmit/atnf/conf/

Widrow, B. \& Stearns, S., 1985, "Adaptive Signal Processing" Prentice Hall. 